\title{
INTERPLANETARY MAGNETIC TAYLOR MICROSCALE AND IMPLICATIONS FOR PLASMA DISSIPATION
}

\author{
W. H. Matthaeus, ${ }^{1}$ J. M. Weygand, ${ }^{2}$ P. Chuychai, ${ }^{1}$ S. Dasso, ${ }^{3}$ C. W. Smith, ${ }^{4}$ and M. G. Kivelson ${ }^{2}$ \\ Received 2007 December 21; accepted 2008 March 24; published 2008 April 21
}

\begin{abstract}
The Taylor microscale, a measure of mean square spatial derivatives, is evaluated for interplanetary magnetic field fluctuations from single- and multiple-point data using Cluster and ACE spacecraft data. The Taylor scale is compared to the measured inner scale, which for hydrodynamics would correspond to the Kolmogorov scale. The results are not consistent with dissipation of the hydrodynamic type, and indicate that solar wind dissipation involves kinetic plasma physics at both proton and electron scales.
\end{abstract}

Subject headings: interplanetary medium — magnetic fields — methods: data analysis — plasmas — solar wind - turbulence

In seeking a balance between relaxation and response to stresses, magnetohydrodynamic (MHD) turbulence evolution (Jokipii \& Coleman 1968; Jokipii 1973; Montgomery et al. 1980; Tu \& Marsch 1995; Goldstein et al. 1995) cascades toward smaller scales, thus amplifying spatial gradients (Monin \& Yaglom 1971-1975). At small scales growth of gradients is arrested, and for a low-collisionality plasma, MHD gives way to kinetic plasma physics, which in some incompletely understood way causes dissipation of MHD fluctuations and plasma heating (see e.g., (Barnes 1979; Leamon et al. 1999; Cranmer 2000; Gary \& Borovsky 2004). Identification of dissipation mechanisms is fundamental in understanding the corona and solar wind, and is of great relevance to astrophysical plasmas in general. Here we examine the small-scale structure of interplanetary magnetic field fluctuations using $A C E$ and Cluster spacecraft data, and study the relationship between two critical length scales - the inner scale which terminates the inertial range at high wavenumber (in viscous hydrodynamics this is the dissipation scale), and the Taylor microscale, related to mean square gradients as described below. The observed relationship between these scales indicates the likely involvement of both proton and electron dynamics in dissipation, and distinguishes the plasma case in an essential way from hydrodynamic dissipation.

In a typical turbulence scenario (Batchelor 1970; Monin \& Yaglom 1971-1975) a power-law inertial range is bounded at long wavelengths by a correlation (outer or energy-containing) scale, and at small scales by a spectral break at the inner scale (Kolmogorov dissipation scale). A third scale, the Taylor microscale, is related to the mean square gradients of velocity or magnetic field, and in hydrodynamics at high Reynolds number (Hinze 1975; Smith et al. 1993; Belmabrouk \& Michard 1998) lies between the inner and outer scales (Monin \& Yaglom 19711975). Less is known about the Taylor scale in low-density astrophysical and space plasmas, and we have neither a theoretical expectation nor, until recently (Matthaeus et al. 2005; Weygand et al. 2007), any observational constraints. The picture is further complicated for a low-density plasma, as kinetic

\footnotetext{
${ }^{1}$ Bartol Research Institute, Department of Physics and Astronomy, University of Delaware, Newark, DE.

${ }^{2}$ Institute for Geophysics and Planetary Physics, University of California, Los Angeles, CA.

${ }^{3}$ Instituto de Astronomía y Física del Espacio (CONICET-UBA) and Departamento de Física (FCEN-UBA), Buenos Aires, Argentina.

${ }^{4}$ Institute for Earth, Oceans, and Space, University of New Hampshire, Durham, NH.
}

scales such as the ion inertial scale (Leamon et al. 1999; Gary $\&$ Borovsky 2004) become influential.

In strong MHD turbulence at high Reynolds numbers the magnetic field and velocity field enter on nearly equal footing. Manifestations of this include the ideal invariance of the cross helicity and the symmetric form taken by the equations in Elsässer representation, and the tendency for near equipartition in the turbulence inertial range (the Alfvén effect) (Kraichnan 1965). Here we focus on solar wind magnetic field fluctuations, mainly because the available spacecraft instrumentation provides higher time resolution magnetic data.

For interplanetary MHD turbulence at 1 AU (Earth orbit) the correlation scale of magnetic field fluctuations ( $\mathrm{Tu} \&$ Marsch 1995; Goldstein et al. 1995) is found to be $\lambda_{c} \approx$ $(1-3) \times 10^{11} \mathrm{~cm}(1 / 100$ to $1 / 50 \mathrm{AU})$ from single-spacecraft data (using the frozen-in flow approximation, for solar wind speed $U \gg u$, characteristic MHD speed $u$ ). Multiple-spacecraft estimation (Matthaeus et al. 2005) gives $\lambda_{c} \approx 1.3 \times 10^{11} \mathrm{~cm}$, or $0.008 \mathrm{AU}$. The magnetic inertial range power-law terminates with subsequent steepening at frequencies near $0.5 \mathrm{~Hz}$ at $1 \mathrm{AU}$. At nominal speed $U \sim 300-500 \mathrm{~km} \mathrm{~s}^{-1}$ this corresponds to spatial scales of $600-1000 \mathrm{~km}$, a few times the ion inertial scale $c / \omega_{p i}$ (Leamon et al. 1998b). This inner scale is often described as a "dissipation scale," in analogy with hydrodynamic terminology. However the kinetic effects that terminate the solar wind inertial range (Barnes 1979; Tu \& Marsch 1995; Gary \& Borovsky 2004) need not be dissipative and include, e.g., dispersive effects as well. To avoid confusion, we refer below to the length associated with the spectral break point as the inner scale without bias as to where in the spectrum dissipation actually occurs.

The hydrodynamic relationships between the scales of interest form an important point of reference. For a typical speed $u=\left(\left\langle|\boldsymbol{v}|^{2}\right\rangle\right)^{1 / 2}$, fluid velocity $\boldsymbol{v}$, enstrophy $\Omega=\left\langle|\boldsymbol{\nabla} \times \boldsymbol{v}|^{2}\right\rangle$, and vorticity $\omega=\sqrt{\Omega}$, let us define the Taylor microscale $\lambda_{\mathrm{T}}$ associated with the velocity field by the relation

$$
\omega^{2}=\frac{u^{2}}{\lambda_{\mathrm{T}}^{2}} .
$$

Here we opt for a simplified definition and therefore we differ by an order-one numerical factor from the standard definitions (Batchelor 1970; Hinze 1975). In the cases discussed below in which the turbulence field of interest is the magnetic field fluctuation $\boldsymbol{b}$ the Taylor scale is analogously defined as $\lambda_{\mathrm{T}}=$ $\left(\left\langle|\boldsymbol{b}|^{2}\right\rangle /\left\langle|\boldsymbol{\nabla} \times \boldsymbol{b}|^{2}\right\rangle\right)^{1 / 2}$. We refer to outer scale (energy containing 


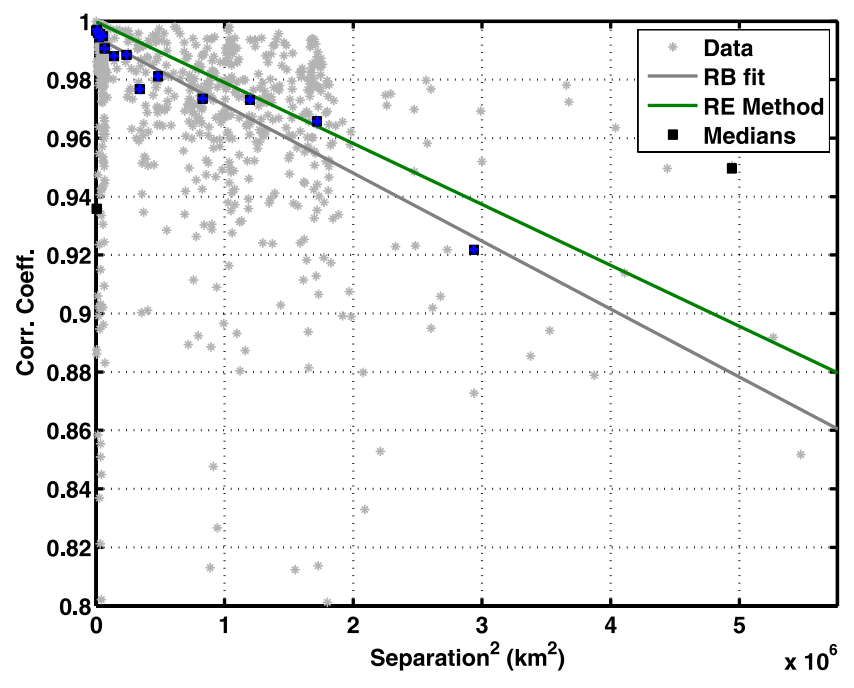

FIG. 1.-Estimates of magnetic autocorrelation function $R(r)$ from Cluster data. Results of extrapolation method and robust fit procedure are shown as lines. Symbols are average correlation values in bins. See Weygand et al. (2007) for details.

scale) $L$, dissipation rate $\epsilon$, and for the viscous case, large-scale Reynolds number $R \equiv u L / \nu$, with viscosity $\nu$. By dimensional analysis the inner scale $\lambda_{d}=1 / k_{d}$, here a true dissipation scale, and its associated wavenumber $k_{d}$ are determined by $k_{d}=$ $1 / \lambda_{d}=\left(\epsilon / \nu^{3}\right)^{1 / 4} \rightarrow k_{d} L=R^{3 / 4}$. The physical interpretation of $k_{d}$ can be made more clear by an alternative derivation. Note that the viscous decay time at wavenumber $k$ is $\tau_{d}=1 /\left(\nu k^{2}\right)$. Define the nonlinear time at wavenumber $k$ as $\tau_{n l}(k)=$ $1 /\left(k u_{k}\right)$ where $u_{k}$ is the speed at $k$. Then assume a steady Kolmogorov spectrum of energy $E(k)=\epsilon^{2 / 3} k^{-5 / 3}$, and notice that $u_{k}=[k E(k)]^{1 / 2}$. It follows that the dissipation wavenumber as defined above solves the equation $\tau_{d}\left(k_{d}\right)=\tau_{\mathrm{nl}}\left(k_{d}\right)$. That is, the dissipation scale is that scale at which dissipation and nonlinear times are equal. In this sense the fluctuations at $k_{d}$ are critically damped.

The hydrodynamical Taylor scale is also related to dissipation. The viscous energy dissipation rate, from the NavierStokes equation, can be expressed as

$$
\epsilon=\nu \Omega=\nu \frac{u^{2}}{\lambda_{\mathrm{T}}^{2}}=\frac{u^{3}}{L} .
$$

The third term suggests a simple interpretation for $\lambda_{\mathrm{T}}$ : concentrating the energy at a single wavenumber, $k_{\mathrm{T}}=1 / \lambda_{\mathrm{T}}$, would not change the instantaneous $\epsilon$, so $\lambda_{\mathrm{T}}$ may be interpreted as the "single-wavenumber equivalent dissipation scale" (Hinze 1975). The final relation above $\epsilon \sim u^{3} / L$ is the von Kármán energy decay estimate for strong turbulence. It follows readily from dimensional analysis as $\epsilon=u^{2} / \tau$ by selecting the timescale $\tau=L / u$. More elegantly, it follows from the assumption of "self-preservation" of the nondimensional correlation functions (von Kármán \& Howarth 1938). Using this, $u L / \nu=$ $\left(L / \lambda_{\mathrm{T}}\right)^{2}$, or, $L / \lambda_{\mathrm{T}}=\sqrt{R}$, and we arrive at

$$
k_{d} \lambda_{\mathrm{T}}=R^{1 / 4}
$$

so that $\lambda_{d}<\lambda_{\mathrm{T}}$ for strong $R \gg 1$ hydrodynamic turbulence.

The Taylor scale also enters in the expansion of the secondorder structure function $S_{2}(r)=\left\langle\left|\boldsymbol{u}-\boldsymbol{u}^{\prime}\right|^{2}\right\rangle=u^{2} \hat{S}_{2}(r)$ for small spatial separations $r$. When $S_{2}$ is regular at the origin,

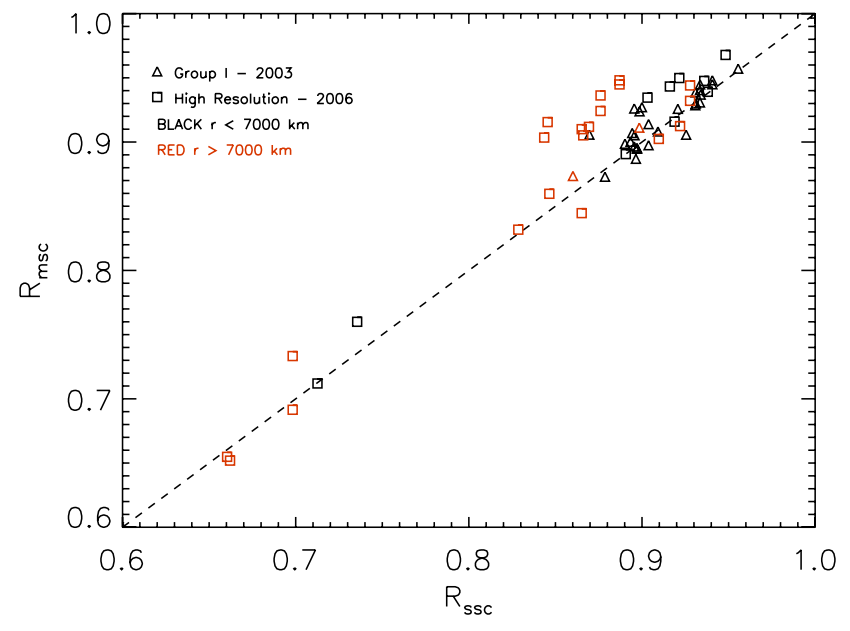

FIG. 2.-Comparison of estimates of the Taylor microscale from singlespacecraft data and from two-spacecraft measurements, using Cluster data. The same intervals are used for each type of determination.

$\hat{S}_{2}(r) \approx 1-r^{2} / \lambda_{\mathrm{T}}^{2}$ for small $r \ll \lambda_{d}$. This is our basis for measurement of the Taylor scale.

Single- and multiple-spacecraft measurements.-Average interplanetary Taylor and correlation scales can be established using simultaneous two-point measurements. Cluster data (2004 January 19-February 2) with separations on the order of 100-200 km indicate (Matthaeus et al. 2005) that $\lambda_{\mathrm{T}}=$ $2478 \pm 702 \mathrm{~km}$. Restricting the determination to data with smaller $r$ in principle gives improved results, but with less available data, statistical uncertainty grows. Richardson extrapolation provides a refined estimate (Weygand et al. 2007), $\lambda_{\mathrm{T}}=2400 \pm 100 \mathrm{~km}$, as illustrated in Figure 1. Here we demonstrate the relationship between the Taylor scale and the inner scale in the interplanetary plasma, employing individual singlespacecraft samples, and the frozen-in flow assumption, converting time separations $\Delta \tau$ to space separations $\Delta r=U \Delta \tau$.

As a preliminary step we demonstrate that correlation estimates from single-spacecraft data are consistent with twospacecraft measurements. The procedure is straightforward. For two-spacecraft values we compute correlation between the signal measured at each spacecraft (Matthaeus et al. 2005), and associate this with the actual spacecraft separation. Next, for the same time period, and for each spacecraft separately, a lagged time-correlation function is computed using frozen-in flow. We then pick out the value that corresponds to the interspacecraft separation, allowing comparison of the two methods. Figure 2 shows that the two methods give very similar results. The data are divided into samples with separations less than or greater than $7000 \mathrm{~km}$. Larger separations show greater discrepancy between single- and multiple-spacecraft correlations. This can be attributed to anisotropy, and to temporal decorrelation during the plasma convection past the spacecraft in the single-spacecraft case. We defer for now a more detailed examination of these issues, and instead make use of this demonstration to bolster our confidence that $\lambda_{\mathrm{T}}$ can be obtained from spacecraft data with a $10 \%-20 \%$ error relative to the more precise two-point multispacecraft determination.

$\lambda_{\mathrm{T}}$ versus $\lambda_{d}$. We now compare estimates of $\lambda_{\mathrm{T}}$ and the inner scale $\lambda_{d}$ obtained from single-spacecraft intervals. Calculation of the inner scale proceeds by computing, for each interval, power-law fits to the spectrum in the inertial range and in the steepened higher wavenumber range (Leamon et al. 
1998a; Smith et al. 2006). The intersection between the fitted curves (straight lines in a log-log representation) defines the spectral "break point," at wavenumber $k_{d}$, and the inner scale $\lambda_{d}=1 / k_{d}$.

Having obtained values of $k_{d}$, we can improve our method for estimating $\lambda_{\mathrm{T}}$. We use magnetic field data at 3 vectors per second. At a nominal $U=400 \mathrm{~km} \mathrm{~s}^{-1}$ this corresponds to a minimum separation $\Delta r_{\text {min }}=U \Delta t_{\min } \approx 125 \mathrm{~km}$. This resolves the spectral break, usually around $0.5 \mathrm{~Hz}$. However there is systematic error in estimating $\lambda_{\mathrm{T}}$ due to unresolved power, which varies with the steepness of the high-frequency spectrum. To address this, for each interval we compute a power-law fit to the high-wavenumber spectrum, assumed to behave as $\sim k^{-\alpha}$ for $k \lambda_{d}>1$. We further assume that this power-law extends to the reciprocal electron inertial scale. This means essentially that any energy not dissipated prior to reaching electron scales will dissipate at the electron scales. Numerical tests show that with minimum separation $\Delta r_{\min }$ as above, an accurate value of $\lambda_{\mathrm{T}}$ is obtained by Richardson extrapolation of $S_{2}(r)$ for cases in which $\alpha>3$.

In the range $3>\alpha>2$, the error in estimation of $\lambda_{\mathrm{T}}$ increases in a calculable fashion. In fact, for every value of $\alpha$, we can estimate a correction factor $\gamma\left(\alpha, \Delta t_{\min }\right)$ to the value of $\lambda_{\mathrm{T}}$ obtained by Richardson extrapolation. Details of this analysis will be given at a later time. Here, for each interval we have a fixed $\Delta t_{\min }$ and an estimate for $\alpha$, so we can compute a corrected Taylor scale $\lambda_{\mathrm{T}}^{\prime}=\gamma \lambda_{\mathrm{T}}$. These values are reported below.

Figure 3 presents results of simultaneous estimation of the inner scale $\lambda_{d}$ and the corrected Taylor scale $\lambda_{\mathrm{T}}^{\prime}$ for 513 intervals of $A C E$ magnetic field data. The reference line indicates $\lambda_{\mathrm{T}}^{\prime}=\lambda_{d}$, which can be viewed as the marginal condition that separates hydrodynamic ordering, $\lambda_{\mathrm{T}}>\lambda_{d}$, and nonhydrodynamic ordering, $\lambda_{\mathrm{T}}<\lambda_{d}$. In hydrodynamic turbulence, all the data would lie below this line (cf. eq. [3] with $R>1$ ). Figure 3 includes 147 magnetic cloud intervals (Smith et al. 2006) and 366 noncloud wind intervals. Interplanetary magnetic clouds (Burlaga et al. 1990) are low plasma beta (pressure/magnetic pressure) intervals that also have lower turbulence levels. They are frequently associated with large structures that may magnetically connect back to the Sun. From Figure 3 we see that most cloud intervals have $\lambda_{\mathrm{T}}<\lambda_{d}$. A few noncloud intervals also lie in this region. In this nonhydrodynamic ordering, the mean square gradient is at scales smaller than the spectral break point scale, and one is led to the conclusion that the plasma dissipation function, whatever its origin, is dissimilar to the familiar viscous-resistive Laplacian form. Now consider the intervals that lie on the hydrodynamic side of the equality. With an estimate $R=230,000$ (Matthaeus et al. 2005), one expects $\lambda_{\mathrm{T}} \approx 20 \lambda_{d}$, but this does not correspond well to the observed distribution. We conclude that even when the samples are hydrodynamically ordered, the relationship expected in high Reynolds number viscous hydrodynamics is not realized.

The result in Figure 3 shows unambiguously that the kinetic processes that terminate the inertial range in the solar wind do not function in the same way as it would with a Laplacian dissipation function and scalar transport coefficients. For viscous hydrodynamics or simple Ohmic-resistive MHD, the inner scale changes in response to the strength of the cascade, producing smaller scale gradients in response to higher Reynolds numbers $(\operatorname{smaller} \nu)$ or larger transfer/dissipation rate $\epsilon$. It has long been recognized (D. C. Montgomery \& M. L. Goldstein 1981 , private communication) that this classical view of dissipation lay on a collision course with the basic physics of the solar wind and other low-density astrophysical plasmas-the

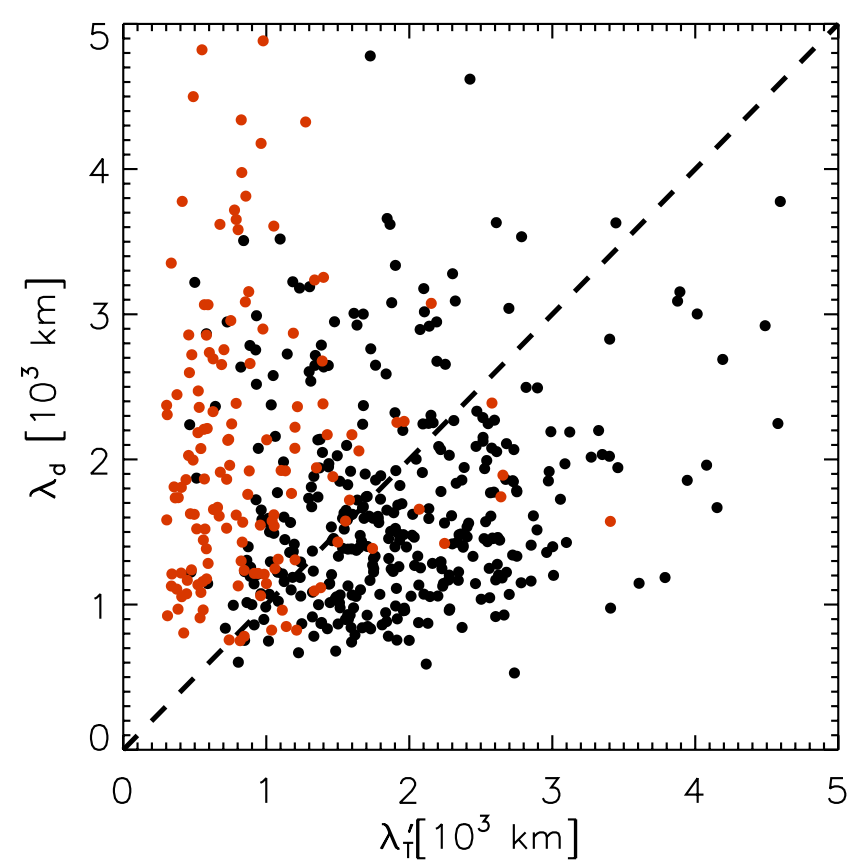

FIG. 3.-Scatter plot of Taylor scale values (from single-spacecraft measurements) and dissipation scale values (defined by the break point at the upper end of the inertial range), from $A C E$ magnetic field data. Red points are magnetic cloud data; black points are other solar wind intervals. The line denotes $\lambda_{\mathrm{T}}=\lambda_{d}$.

plasma mandates the relevance of certain kinetic length scales, the proton gyroradius, proton inertial scale, etc. These will control the onset of dissipation and dispersion, and the termination of the inertial range may therefore behave rather differently than in hydrodynamics. Furthermore these length scales are controlled by bulk plasma parameters, density, average magnetic field strength, and temperature, and are not regulated in any direct way by the turbulence cascade. While turbulence ultimately contributes to control of some of these quantities, it seems certain that the simple Batchelor-Kolmogorov perspective embodied in equation (3) cannot survive. Recent evidence (Smith et al. 2006) supports the plasma dissipation picture, as no clear relation is found between the spectral break point $\left(1 / \lambda_{d}\right)$ and values of $\epsilon$ estimated from inertial range power levels.

Clues regarding what replaces the standard hydrodynamic picture of dissipation are present in Figure 3 (see also Smith et al. 2006). Evidently in magnetic clouds the gradients can increase so much that the Taylor scale becomes smaller than the spectral steepening scale $\left(\lambda_{d}\right)$, and this occurs to a lesser extent in noncloud intervals of solar wind. Given that $\lambda_{d}$ is of the order of the ion inertial scale, one concludes that electron dynamics becomes relatively more important in clouds. Many possible electron processes would be candidates to explain this; see, e.g., Dasso et al. (2003). This characterization also applies to a minority of noncloud intervals. At present it is not entirely clear how the plasma differentiates between cases in which the small-scale gradients will be limited at (or longer than) proton scales, or when saturation of gradients involves electron dynamics, possibly even at the scales as small as the electron inertial scale. What is clear is that the growth of magnetic gradients is sometimes arrested near the proton scales, and sometimes at much smaller scales, perhaps as small as electron scales. Figure 4 gives further insight concerning regulation of 


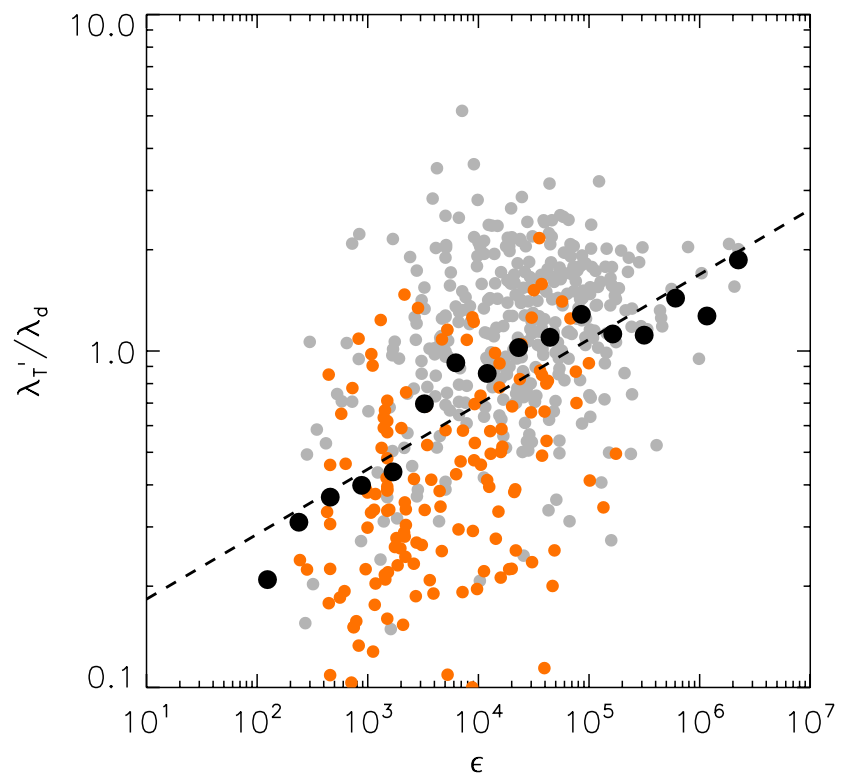

FIG. 4.-Scatter plot of the ratio of Taylor scale/dissipation scale vs. $\epsilon=$ estimate of the cascade rate in the inertial range. The data are binned and the best power-law fit is $\lambda_{\mathrm{T}}^{\prime} / \lambda_{d}=\left(\epsilon / \epsilon_{0}\right)^{0.193} \sim \delta b^{0.579}$ for fit constant $\epsilon_{0}$, since $\epsilon \sim$ $\delta b^{3}$. See Smith et al. (2006).

the small-scale gradients. There is a suggestion (see caption) of a positive correlation between $\epsilon$ and $\lambda_{T} / \lambda_{d}$. This indicates that a stronger cascade favors limitation of the magnetic gradients near proton scales, while a weaker cascade favors gradients at smaller scales. The former suggests greater involvement of proton dynamics in stronger turbulence. For a weaker cascade, the gradients move toward higher wavenumber until limited by other processes, presumably due to electron dynamics.

Conclusions.-At first the above results may seem counterintuitive - in the classical hydrodynamic picture a stronger cascade generates smaller scale dissipation. However, in plasma physics, many kinetic processes including instabilities are associated with characteristic scales (e.g., resonances) but may not be triggered until some threshold condition is satisfied. The above results are consistent with a picture in which steeper gradients associated with stronger cascade activate additional channels of dissipation at ion scales. Conversely with weaker transfer rates, the protons see more gentle gradients and respond less vigorously, dissipating less of the available flux of cascaded energy. Consequently, for weaker cascade rates, more energy appears in the scales between the proton and electron inertial scales, where eventually additional (and electron-related) mechanisms provide dissipation. The above results highlight the nonhydrodynamic nature of dissipation in the solar wind, adding some new perspective on the relationship between mean square gradients and dissipation in a turbulent low-collisionality plasma. The role of spectral anisotropy and other factors, such as proton and electron plasma beta, as well as details of the kinetic processes involved remains to be determined in future studies.

This research supported in part by NASA (RSSW1AU and Cluster GI programs) NAG5-8134 and NAG5-11603, and NSF-ATM 0539995. C. W. S. is supported by JPL contract PC251459 (NASA NAG5-6912 for the ACE MAG instrument). S. D. is a member of CIC (CONICET) and is partially supported by grants PIP 6220 (CONICET) and PICT 03-33370 (ANPCyT).

\section{REFERENCES}

Barnes, A. 1979, in Solar System Plasma Physics, Vol. 1, edited by E. N. Parker, C. F. Kennel, \& L. J. Lanzerotti (Amsterdam: North-Holland), 249

Batchelor, G. K. 1970, Theory of Homogeneous Turbulence (Cambridge: Cambridge Univ. Press)

Belmabrouk, H., \& Michard, M. 1998, Exp. Fluids, 25, 69

Burlaga, L. F., Lepping, R. P., \& Jones, J. A. 1990, in Physics of Magnetic Flux Ropes, ed. C. T. Russell et al. (Geophys. Monogr. 58; Washington: AGU), 373

Cranmer, S. R. 2000, ApJ, 532, 1197

Dasso, S., Gratton, F. T., \& Farrugia, C. J. 2003, J. Geophys. Res., 108, 1149

Gary, S. P., \& Borovsky, J. E. 2004, J. Geophys. Res., 109, 6105

Goldstein, M. L., Roberts, D. A., \& Matthaeus, W. H. 1995, ARA\&A, 33, 283

Hinze, J. O. 1975, Turbulence (New York: McGraw Hill)

Jokipii, J., \& Coleman, P. 1968, J. Geophys. Res., 73, 5495

Jokipii, J. R. 1973, ARA\&A, 11, 1

Kraichnan, R. H. 1965, Phys. Fluids, 8, 1385

Leamon, R. J., Matthaeus, W. H., Smith, C. W., \& Wong, H. K. 1998a, ApJ, 507, L181
Leamon, R. J., Smith, C. W., Ness, N. F., \& Matthaeus, W. H. 1998b, J. Geophys. Res., 103, 4775

Leamon, R. J., Smith, C. W., Ness, N. F., \& Wong, H. K. 1999, J. Geophys. Res., 104, 22331

Matthaeus, W. H., Dasso, S., Weygand, J. M., Milano, L. J., Smith, C. W., \& Kivelson, M. G. 2005, Phys. Rev. Lett., 95, 231101

Monin, A. S., \& Yaglom, A. M. 1971-1975, Statistical Fluid Mechanics (Cambridge: MIT Press)

Montgomery, D. C., et al. 1980, Report of the NASA Plasma Turbulence Explorer Study Group (Pasadena: NASA/JPL)

Smith, C. W., Hamilton, K., Vasquez, B. J., \& Leamon, R. J. 2006, ApJ, 645, L85

Smith, M. R., Donnelly, R. J., Goldenfeld, N., \& Vinen, W. F. 1993, Phys. Rev. Lett., 71, 2583

Tu, C.-Y., \& Marsch, E. 1995, Space Sci. Rev., 73, 1

von Kármán, T., \& Howarth, L. 1938, Proc. R. Soc. London A, 164, 192

Weygand, J. M., Matthaeus, W. H., Dasso, S., Kivelson, M. G., \& Walker, R. J. 2007, J. Geophys. Res., 112, A10201 